\title{
Influence of cavity depth on marginal degradation of amalgam restorations
}

\author{
Asbjørn Jokstad \\ Department of Anatomy, School of Dentistry, University of Oslo, Oslo, and NIOM, \\ Scandinavian Institute of Dental Materials, Haslum, Norway
}

Jokstad A. Influence of cavity depth on marginal degradation of amalgam restorations. Acta Odontol Scand 1991;49:65-71. Oslo. ISSN 0001-6357.

\begin{abstract}
In a 5-year clinical trial it was observed that the marginal degradation of class-II amalgam restorations could be related to the bulk of the restoration. The association between the occlusal cavity depth and the marginal degradation was observed after 6 months and varied for the different types of alloy. Ridit scores of the marginal degradation were correlated to various indices of the cavity sizes, to assess the possible reason for this association. The possibilities of the relationship being an indirect effect caused by longer condensation times or by poorer condensation due to the use of larger condenser sizes were rejected. Furthermore, the possibility that the association was the result of potential buildup of stresses on the restoration margins caused by flexible cusps and axial walls was not apparent. A possible mechanism may be that marginal degradation is the result of short-term or long-term expansion, or even extrusion of amalgam. Expansion may theoretically be caused over short periods by temperature changes or over long periods by corrosion or phase shifts in the amalgam. The theory does not exclude the role of creep or corrosion and may furthermore explain the lack of correlation between in-vitro tests and in-vivo performance of amalgam restorations. $\square$ Clinical study; dental materials; expansion; marginal ditch; operative dentistry
\end{abstract}

Asbjørn Jokstad, Department of Anatomy, University of Oslo, P.O.B. 1052, Blindern, 0316 Oslo 3, Norway

In a 5-year clinical trial it was observed that the marginal degradation of class-II amalgam restorations seemed to be influenced by the depth of the cavity preparations (1). This observation was somewhat surprising and warranted further evaluations. It was postulated that four possible mechanisms uld explain the phenomenon. First, when deep or large cavities are restored, the operators use larger condensation instruments, resulting in less condensation force and lack of adaptation, porosities, and poorer physical properties of the amalgam $(2,3)$. This theory would be supported if the marginal degradation could be related also to the volume of the cavities. A second possible mechanism is that the longer time needed to fill deeper, or larger, cavities would result in less-than-optimal occlusal top layers of amalgam, compared with the amalgam placed in shallow or small cavities $(2,3)$. If the time factor for condensing the amalgam is impor- tant, the differences between the large and small restorations would be especially obvious for the fast-set alloys. A third proposed mechanism is that the masticatory forces induce stresses at the restoration margins in the cavities with flexible axial walls and cusps (4-8). If this biomechanical mechanism is correct, it was assumed that the margins would show increased degradation in cavities in which high cusp movements are possiblethat is, in cavities with deep or wide occlusal parts and with cavity walls diverging in the occlusal direction. The last potential mechanism is a possible dimensional instability of amalgam, resulting in an expansion related to the thickness of the restoration. If expansion of amalgam is the cause of marginal degradation, a variation in the kinetics and degree of degradation as a function of type of alloys could be expected, regardless of the actual cause of the expansion. The different analyses aimed to investigate the most prob- 

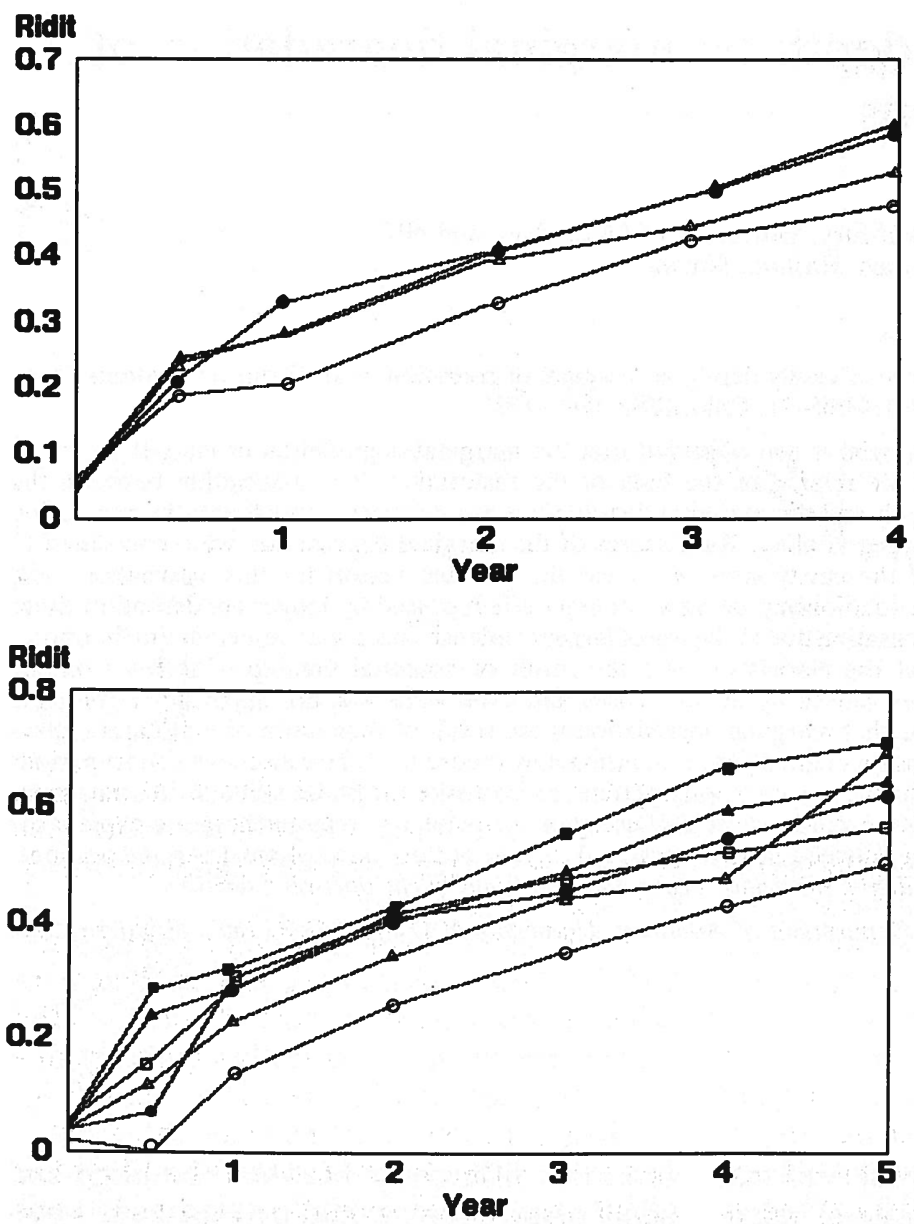

Fig. 1. The ridit means for the restorations placed in large cavities (circles) and small cavities (triangles), made from a fast-setting amalgam $T$ (Tytin: open symbols; $n=59$ and $n=$ $15)$ and a slowly setting amalgam (Amalcap: closed symbols; $\boldsymbol{n}=$ 57 and $n=25$ ). No individual paired comparisons reach the required critical normal curve value of 2.6 to be at a significance level of $\alpha=0.05$, according to the Bonferroni criterion.

Fig. 2. The ridit means for the restorations placed in cavities with shallow (circles: less than $2 \mathrm{~mm}$ ), medium (triangles), or deep occlusal depth (squares: more than $3 \mathrm{~mm}$ ), made from a fast-setting amalgam $\mathrm{T}$ (Tytin: open symbols; $n=16, n=46$, $n=13$, respectively) and a slowly setting amalgam (Amalcap: closed symbols; $n=$ $18, n=54, n=10$, respectively). No individual paired comparisons reach the required critical normal curve value of 2.9 to be at a signifcance level of $\alpha=0.05$, according to the Bonferroni criterion. able mechanism for the relationship between the marginal degradation and the thickness of the restorations.

\section{Materials and methods}

The material comprised 468 class-II amalgam restorations placed by 7 clinicians in 210 patients. An impression was made of the prepared cavity before the tooth was restored with amalgam (Xantopren blue and Optosil, Bayer, Leverkusen, Germany). Casts were made from the impressions within $72 \mathrm{~h}$, using an epoxy material (Durcupan, Fluka AG, Buchs, Switzerland), and examined in a stereomicroscope (Spencer Ameri- can Optical) at $\times 10$ and $\times 20$ (9). Five different alloys were used: Revalloy (SS White Ltd., U.K.), Indiloy (Shofu Dental Corp., Japan), Tytin (SS White Ltd., U.K Dispersalloy (Johnson \& Johnson, USA), and Amalcap Non-gamma 2 (Vivadent, Germany). Impressions of the restorations were made each year over 5 years. The marginal degradation of the restorations was scored in accordance with a 6-point ordinal scale, and related to features of the cavity preparations. Statistical analysis of the marginal degradation was performed by transforming the categorical values to ridit scores and by using paired comparison tests utilizing the Bonferroni correction factor. The materials and methods have been described in detail previously $(1,9,10)$. 
Fig. 3. The ridit means for the restorations placed in cavities with shallow (circles: less than $2 \mathrm{~mm}$ ), medium (triangles), or deep occlusal depth (squares: more than $3 \mathrm{~mm}$ occlusal depth) as a function of the axial cavity walls converging occlusally (open symbols, $n=46, n=175, n=$ 41 , respectively) or axial cavity walls diverging occlusally (closed symbols, $n=43, n=119, n=$ 15 , respectively). The numbers at the parallel lines indicate the critical ratios between the mean ridits. Each individual paired comparison requires a critical normal curve value of 2.9 to be at a significance level of $\alpha=$ 0.05 , according to the Bonferroni terion.

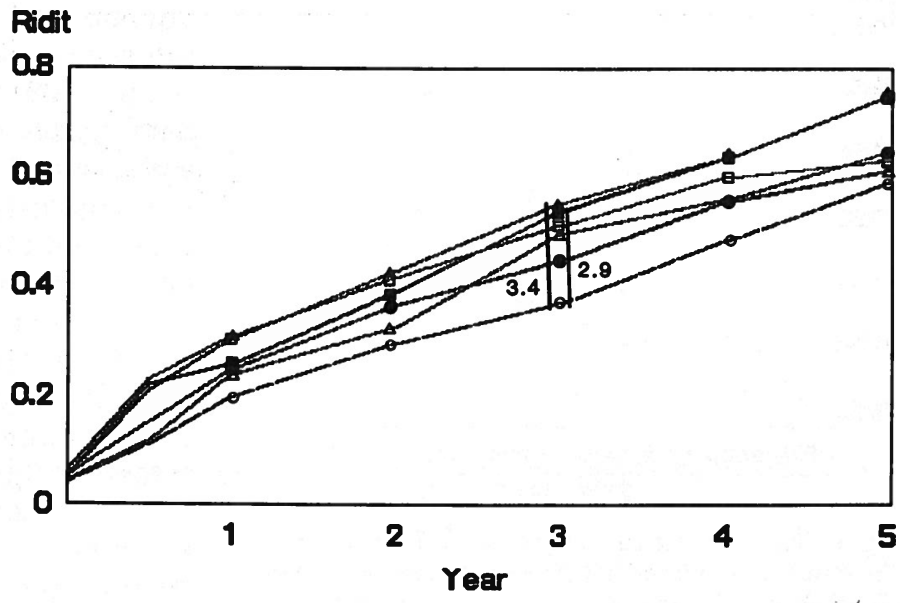

\section{Results}

The ridit values of the restorations placed in large and voluminous cavities were compared with the ridit values of the restorations in small cavities (Fig. 1). There was no relationship between the volume of the cavity and the marginal degradation for either type of alloy. On the other hand, the marginal degradation and the occlusal cavity depth was related, and this was apparent both for the fast-setting and the slowly setting alloys (Fig. 2). The association between the cavity

Ridit

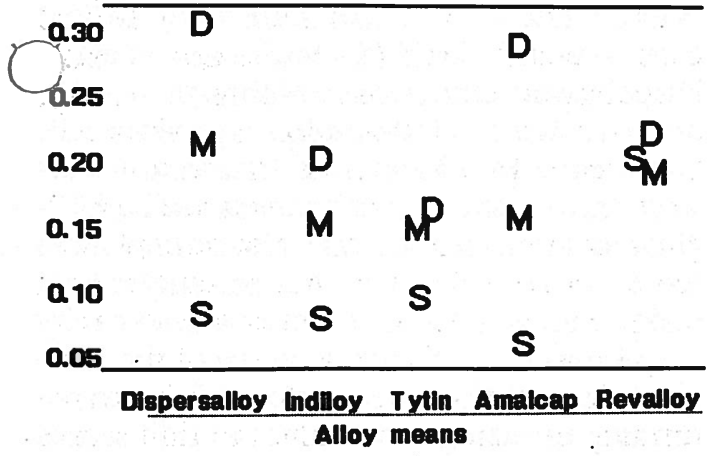

Fig. 4. The ridit means, varying from 0.05 to 0.30 , of the restorations placed in cavities with shallow $(<2 \mathrm{~mm}$, S), medium (M), and deep (>3 mm, D) occlusal depth made by 5 different alloys. Results after 6 months. depth and the marginal degradation was seen after 6 months, whereas thereafter the ridit curves were relatively parallel during the next 5 years.

When the restorations were categorized both by the occlusal depth and by the cavity walls' convergence or divergence in the occlusal direction, statistically significant differences could be observed at 3 years (Fig. 3 ). When the restorations were categorized by the occlusal depth and width and by the cavity walls' convergence, the ridit scores of the subgroups were identical.

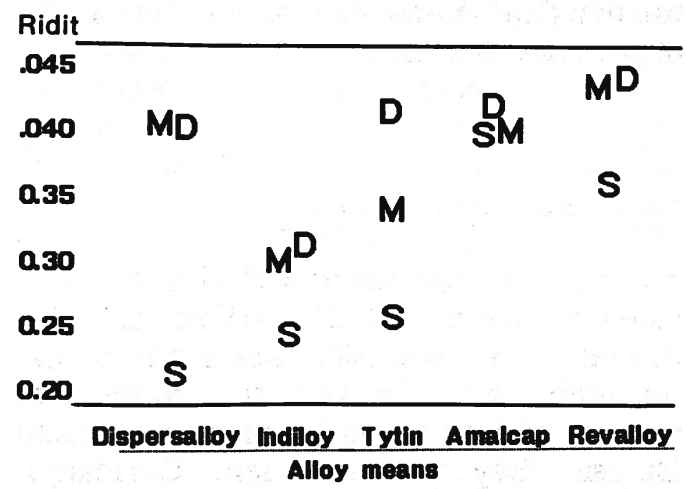

Fig. 5. The ridit means, varying from 0.20 to 0.45 , of the restorations placed in cavities with shallow $(<2 \mathrm{~mm}$, S), medium (M), and deep (>3 mm, D) occlusal depth made by 5 different alloys. Results after 2 years. 


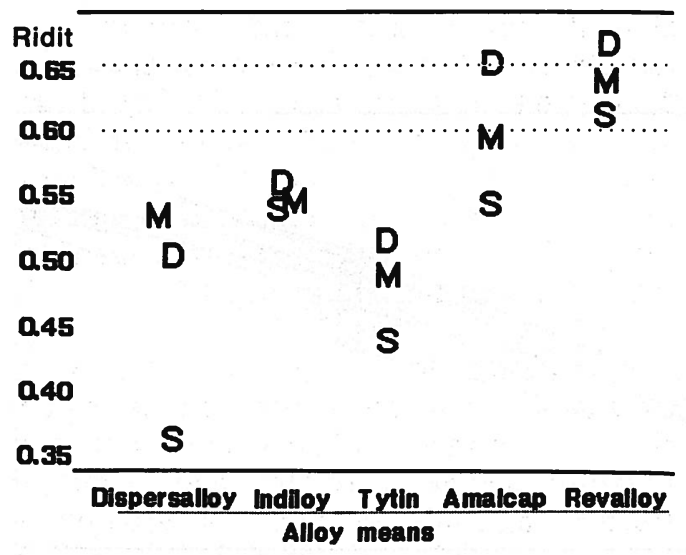

Fig. 6. The ridit means, varying from 0.35 to 0.65 , of the restorations placed in cavities with shallow $(<2 \mathrm{~mm}$, S), medium (M), and deep (>3 mm, D) occlusal depth made by 5 different alloys. Results after 4 years.

The relationship between the marginal degradation and the occlusal cavity depths varied slightly with the different types of alloys and over time (Figs. 4-6). The association between marginal degradation and cavity depth was most marked for Dispersalloy but was almost absent for Revalloy.

\section{Discussion}

The time factor is probably an important element for understanding the mechanism of marginal degradation. It was noted that the relationship among the alloys, the cavity depth, and the marginal degradation was seen after 6 months, whereas thereafter the ridit curves were relatively parallel during the next 5 years (Figs. 1, 2, and 4). The observation may signify that the major part of clinically observable marginal degradation or of unobservable microstructural and substructural stresses in the margins develop during the 1st year after placement of the restoration. This theory may explain why in some clinical trials the investigators report alloy-dependent differences after relatively short observation periods but no further discrimination between marginal scores of alloys during the next few years of observation $(10-12)$.
The lack of association between the cavity volume and the marginal degradation was interesting since it could be postulated that the operators would use larger condensation instruments when filling large cavities than when filling small cavities, with subsequent less condensation pressure. Lack of good condensation is one among several manipulative variables that may influence the mercury content and the morphologies and volume fractions of the different phases $(13$, 14). Thus the lack of relationship to the cavity volume but not to cavity depth indicates that the effect of the condensation time or the condensation pressure cannot explain the observed increased degradation in the deep cavities.

The size of the cavity was calculated as.

$$
\begin{aligned}
& \text { CW(average)*CW(isthmus)*CW(dovetail) } \\
& 3 \\
& { }^{*} \text { Depth*MD }+\frac{P C\left(\text { isthmus) }{ }^{*} \mathrm{PC}(\text { gingiva })\right.}{2} \\
& \text { depth*MR-GM }
\end{aligned}
$$

where $\mathrm{CW}=$ occlusal cavity width, $\mathrm{PC}=$ proximal buccolingual extension, $\mathrm{MD}=$ mesiodistal extension, and MR-GM = distance between the marginal ridge and gingival margin.

Higher ridit scores were observed for the restorations placed in cavities with axial walls diverging occlusally than in the other cavities (Fig. 3). The increase was especially marked in the cavities with remaining thin flexible cusps-that is, cavities with large occlus buccolingual extensions. Although the diferences between the subgroups were not statistically significant, the data partly support the biomechanical mechanism (4-7). However, the increase may also be explained by the low amalgam margin angles frequently present under these conditions (1517). Moreover, although the depth of the occlusal part is known to affect the strength of the remaining tooth, the association between the marginal degradation and the cusp rigidity disappeared when the occlusal cavity depth was included as a further categorization criterion. The observations in 
the present study therefore do not show whether or how a biomechanical mechanism plays a role in the marginal degradation of amalgam restorations.

The third potential explanation of the relationship between the bulk of the restoration and marginal degradation is a possible short- or long-term dimensional instability of amalgam. This amalgam expansion would be different from the well-known expansion of zinc-containing amalgams, resulting from contamination of certain solutes with specific $\mathrm{pH}$ values. A close relationship between marginal degradation and extrusions of the proximal and the occlusal parts of the restorations was observed in two linical trials for three different types of alloy 18, 19). Extrusion of amalgam was also indirectly registered as an increased frequency of the score 'Catch of explorer towards restoration' compared with the base-line evaluation in another 4-year trial (20). However, reports of observations in clinical trials of a possible extrusion of amalgam restorations are sparse $(21,22)$. Furthermore, among several clinical in-vivo wear studies of dental materials only two have reported a negative wear of amalgam restorations - that is, extrusion of the restorations $(23,24)$. Comparisons of the wear rates in the latter studies are, however, difficult because of the great variation of the measuring techniques and the presentation of the results. Finally, only one authoritative dental material textbook states that amalgam continuously expands due to the corrosion, but the abrasive forces constantly reduce the eclusal surfaces (25). Unfortunately, the authors fail to present references to support this statement.

In-vitro studies have shown that amalgam may undergo a phase transformation shift of gamma-1 to beta-1 (26), gamma-2 to eta (27), and beta-1 to alpha-1 (28). These phase changes induce voids (29), change of the mechanical properties (30), and expansion $(31,32)$. When the short- and long-term expansion of unrestricted and restricted cylindrical amalgam specimens are compared, the latter expand more lengthwise (33-35), and the speed and degree of expansion were shown to be related to the type of alloy (35).
Both the rate of phase transformation and the change of creep have been shown to depend on the temperature $(36-38)$. Thus, the data from the in-vitro studies indicate that there may be a possible association between the phase transformations and the creep values, and a relationship to the marginal degradation has been suggested (35, $39,40)$.

In addition to the potential long-term expansion, short-term intermittent expansion and contraction of amalgam due to temperature changes may also lead to fatigue fractures and subsequent degradation of the margins $(41,42)$. Although it is uncertain which is the more important, or even correct, the two alternative mechanisms explaining the association between the restoration bulk and the marginal degradation are not contradictory.

The many suggested mechanisms for the marginal degradation of amalgam restorations may seem to conflict $(43,44)$, but an explanatory model may interrelate the various theories: marginal degradation is the result of dimensional instability caused by corrosion, temperature changes, diffusion and reallocation of mercury, and transformation of the different phases of the matrix. To what extent the expansion will lead to fatigue fracturing, microcracking, or extrusion is also related to the roughness of the cavity wall, the adaptability of the specific alloy, and the mechanical properties of the alloys. The creep and corrosion behavior of freshly mixed amalgams will only give imprecise long-term indications since these properties change continuously with the phase shifts (45) and loading stresses (46). The factors that have been found to influence these properties also affect the long-term dimensional stability of amalgam. The different clinical behavior of restorations made from various alloys with practically identical creep and corrosion properties may be caused by a different interand intra-crystalline location of $\mathrm{Sn}, \mathrm{Zn}$, or impurities in the matrix, factors that are known to be connected with the long-term phase shifts $(31,47,48)$. If expansion of amalgam occurs intra-orally, the extrusion would be observable at all margins. 
However, proximal degradation is difficult to record clinically, and only one study has so far been published in which a positive correlation between the occlusal and the proximal degradation was observed (49). The influence of the cavity depth on marginal degradation may explain the conflicting conclusions in reports in which the marginal degradation has been correlated to the buccolingual cavity widths $(2,50)$, because when a cavity is extended buccolingually, the cavity depth usually, but not always, also increases. Furthermore, the model may explain why the marginal leakage is less for restorations placed in cavities with rough cavity walls than with smooth walls (51). In addition, the theory is not in conflict with any results from marginal leakage tests (52). Finally, the model may explain the dissimilarities of the morphologic appearances of early marginal ditching seen, for example, between the well-adapted Tytin compared with the less well adapted Dispersalloy (53).

\section{References}

1. Jokstad A, Mjör IA. Cavity design and marginal degradation of the occlusal part of class-II amalgam restorations. Acta Odontol Scand 1989;48:389-97.

2. Osborne JW, Gale EN. Failure at the margin of amalgams as affected by cavity width, tooth position and alloy selection. J Dent Res 1981;60:681-5.

3. Berry TG, Laswell HR, Osborne JW, Gale EN. Width of isthmus and marginal failure of restorations of amalgam. Oper Dent 1981;6:55-8.

4. Granath LE, Hiltscher R. Strength of edge of class II cavity restorations in relation to buccolingual shape of cavity. Odont Rev 1970;21:189-97.

5. Derand T. Marginal failure of amalgam class II restoration. J Dent Res 1977;56:481-5.

6. Peters MC, Poort HW. Biomechanical stress analysis of the amalgam-tooth interface. J Dent Res 1983;62:358-62.

7. de Vree JH, Peters MC, Plasschaert AJ. The influence of modification of cavity design on distribution of stresses in a restored molar. J Dent Res 1984; 63:1217-20.

8. Williams PT, Cahoon JR. Amalgam margin breakdown caused by creep fatigue rupture. J Dent Res 1989;68:1188-93.

9. Jokstad A, Mjör IA. Cavity designs for class II amalgam restorations. Acta Odontol Scand 1987;45: 257-73.

10. Jokstad A, Mjör IA. Clinical variables affecting the marginal degradation of amalgam restorations. Acta Odontol Scand 1989;48:379-87.

11. Larson TH, Sabott D, Cooley R, Greener EH.
A clinical study of marginal integrity and tarnish behaviour of three $\mathrm{Cu}$-rich amalgam systems. J Oral Rehabil 1979;6:61-7.

12. Ricker JB, Greener EH. Early observations and three-year clinical evaluation of four amalgam alloys. Oper Dent 1988;13:119-27.

13. Jørgensen Dreyer $K$. Dentale amalgamer. 2nd ed. Copenhagen: Odontologisk Boghandels Forlag, 1976.

14. Sarkar NK. Creep, corrosion and marginal fracture of dental amalgams. J Oral Rehabil 1978;5:413-23.

15. Elderton RJ. Cavo-surface angles, amalgam margin angles and occlusal cavity preparations. Br Dent J 1984;156:319-24.

16. Mathewson RJ. Determination of cavo-surface angles in primary molar cavity preparations. J South Calif Dent Assoc 1972;40:1062-6.

17. Machida Y, Imanishi T, Yakushin M. Study of the marginal fracture of amalgam restorations in deciduous teeth. Shikwa Gakuho 1969;69:92-6.

18. Mahler DB, Van Eysden J. Occlusal extrusion clinical amalgam restorations. IADR Program and abstracts of papers, No. 26, 1974.

19. Terkla LG, Mahler DB, Van Eysden J. Analysis of amalgam cavity design. J Prosthet Dent 1973;29: 204-9.

20. Leidal TI, Dahl JE. Marginal integrity of amalgam restorations. Acta Odontol Scand 1980;38:81-8.

21. Sweeney JT. Amalgam manipulation manual vs mechanical aids. II. Comparison of clinical applications. J Am Dent Assoc 1940;27:1940-9.

22. Vrijhoef MA, Spanauf AJ, Driessens FC. Excessive long-term dimensional change of dental amalgam. Aust Dent J 1975;20:37-8.

23. Roulet JF, Mettler P, Friedrich K. Ein klinischer vergleich dreier komposits mit amalgam fur klassII-fullungen under besonderer berucksichtigung der abrasion. Schweiz Monatschr Zahnmed 1980;90: $18-30$.

24. Mettler P, Friedrich U, Roulet JF. Studie über abrasion von amalgam und komposits im sitenzahnbereich. Schweiz Monatsschr Zahnmed 1978;88:324-44.

25. Leinfelder KF, Lemons JE. Clinical restorative materials and techniques. Philadelphia: Lea \& Febiger, 1988:26.

26. Johnson LB Jr. X-ray diffraction evidence for th presence of beta $(\mathrm{Ag}-\mathrm{Hg})$ in dental amalgam. J Biomed Mater Res 1967;1:285-98.

27. Marshall SJ, Marshall GW Jr. Time-dependent phase changes in Cu-rich amalgams. J Biomed Mater Res 1979;13:395-406.

28. Reynolds CL Jr. The redistribution of mercury as observed in the phase transformations in amalgams. J Biomed Mater Res 1973;7:335-52.

29. Abbott JR, Makinson OF. Removal of gamma-2phase in amalgam. J Biomed Mater Res 1979;13: 790-5.

30. Espevik S. Creep of dental amalgam as a function of heat treatment. Scand J Dent Res 1975;83:3824.

31. Rutz AC. Amalgam delayed expansion and acid treatment of fillings. J Dent Res 1970;49:1172. 
32. Jënsen SJ, Jørgensen Dreyer K. Dimensional and phase changes of dental amalgams. Scand J Dent Res 1985;93:351-6.

33. Fischer $\mathbf{C H}$, Lewald $\mathrm{H}$. Neuartige untersuchungen über die dimensionsänderungen der amalgame wahrend der erhätung. Dtsch Zahn Mund Kieferhkd 1954;20:463-80.

34. Jørgensen Dreyer K, Holst K. Setting expansion of amalgam influenced of the direction of condensation. Acta Odontol Scand 1964;22:201-6.

35. Paffenbarger GC, RuppNW, Patel PR. Dimensional change of dental amalgamandasuggested correlation between marginal integrity and creep. J Am Dent Assoc1979;99:31-37.

36. Jørgensen Dreyer K, Cristiansen I, Esbensen AL. Dentale amalgamers merkuroskopiske ekspansion. Tandlaegebl1973;77:389-97.

37. Vrijhoef MA, Driessens FC. Long term phase changes in dental amalgam after setting. J Biomed Mater Res 1974;8:435-42.

. Espevik S. Creep and phase transformation in dental amalgam. J Dent Res 1977;56:36-9.

39. Osborne JW, Winchell PG, Phillips RW. A hypothetical mechanism by which creep causes marginal fracture of amalgam restorations. J Indiana Dent Assoc 1978;57:16-7.

40. Beech DR. High copper alloys for dental amalgam. Int Dent J 1982;32:240-51.

41. Williams PT, Hedge GL. Creep-fatigue as a possible cause of dental amalgam margin failure. J Dent Res 1985;64:470-5.

42. Wright KW, Yettram AL. Finite element stress analysis of a class I amalgam restoration subjected to setting and thermal expansion. J Dent Res 1978;57:715-23.

43. Bryant RW. Marginal fracture of amalgam restorations. A review. I. Aust Dent J 1981;26:162-6.

44. Bryant RW. Marginal fracture of amalgam restorations. A review. II. Aust Dent J 1981;26:2224.

45. Espevik S. Creep of dental amalgam and its phases. Scand J Dent Res 1977;85:492-5.

46. Williams KR. cyclic creep and fracture of dental amalgam. Biomaterials 1983;4:255-261.

47. Johnson LB Jr, Paffenbarger GC. The role of zinc in dental amalgam. J Dent Res 1980;59:1412-9.

48. Abbott JR, Miller DR, Netherway DJ. Influence of alloy composition on the hardening of silver-tin dental amalgam. J Biomed Mater Res 1986;20: 1391-1400.

49. Goldberg GJ, Tanzer J, Munster E, Amara J, Thal F, Birkhed D. Cross-sectional clinical evaluation of recurrent caries. Restoration of marginal integrity and oral hygiene status. J Am Dent Assoc 1981; 102:635-41.

50. Mahler DB, Marantz RL. Marginal fracture of amalgam. Effect of type of tooth and restoration class and size. J Dent Res 1980;59:1497-1500.

51. Kidd EA. Microleakage-a review. J Dent 1976; 4:199-206.

52. Grieve AR. Marginal adaptation of amalgam in relation to the finish of cavity margins. Br Dent $\mathrm{J}$ 1971;130:239-42.

53. Marker VA, McKinney TW, Filler WH, Miller BH, Mitchell TG, Okabe T. A study design for an in vivo investigation of marginal fracture in amalgam restorations. Dent Mater 1987;3:322-30. 


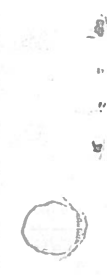

( $)$ 